\title{
Intra-Arterial Vasopressin in Post-Pancreatectomy Hemorrhage: Re-Familiarizing Current Surgical Trainees With a Once Novel Technique
}

\author{
Nikita N. Machado ${ }^{\mathrm{a}}$, Terence Jackson ${ }^{\mathrm{b}}$, Akilan Arumugham ${ }^{\mathrm{c}}$, \\ D. Rohan Jeyarajah ${ }^{\text {b, d, e }}$
}

\begin{abstract}
Post-pancreatectomy hemorrhage (PPH) is known to be a significant cause of post-operative morbidity. In this article we discuss the utility of intra-arterial vasopressin infusion in controlling PPH that is not amenable to more traditional methods of endoscopic or interventional radiology (IR) interventions. While not considered one of the first-line modalities, intra-arterial vasopressin finds a role in specific cases; and our aim is to familiarize current surgical trainees with this treatment modality, which can prove invaluable in challenging clinical situations.
\end{abstract}

Keywords: Post-pancreatectomy hemorrhage; Intra-arterial vasopressin; Education

\section{Introduction}

Pancreatic surgery can be associated with significant morbidity. One of the most serious complications associated with these operations is that of post-pancreatectomy hemorrhage (PPH). Since the severity and outcomes from PPH can be fairly variable, the International Study Group of Pancreatic Surgery (ISGPS) compiled a more objective and standardized classification of post-pancreatectomy bleeding. The codification is based on both a consensus of expert clinical experience and a

Manuscript submitted August 10, 2020, accepted August 19, 2020

Published online June 28, 2021

aDepartment of Surgery, University Hospitals Conneaut Medical Center, Conneaut, $\mathrm{OH}, \mathrm{USA}$

${ }^{b}$ Department of Surgery, Methodist Richardson Medical Center, Richardson, TX, USA

'Department of Radiology, Methodist Richardson Medical Center, Richardson, TX, USA

dTCU/UNTHSC School of Medicine, Fort Worth, TX, USA

${ }^{e}$ Corresponding Author: D. Rohan Jeyarajah, Department of Surgery, Methodist Richardson Medical Center, Richardson, 2805 East President George Bush Highway, Richardson, TX 75082, USA. Email: drj@tscsurgical.com

doi: https://doi.org/10.14740/jcs420 review of the existing literature.

There are multiple reasons for bleeding post pancreatectomy, as well as a myriad of different techniques that can be used for the management of PPH, alone or in combination with one another. These include endoscopic therapy, endovascular interventions and surgery.

This article is an attempt to re-familiarize the current generation of surgical trainees and physicians with the use of intra-arterial vasopressin infusion in the treatment of PPH. First described in 1968 for variceal bleeding, this treatment modality has fallen out of favor. Due to advances in the field of interventional radiology (IR), vasopressin infusion has been largely replaced with targeted embolization of culprit vessels. However, vasopressin infusion remains an important tool in our arsenal for treating PPH, especially in those cases where the bleed can be visualized but is inaccessible/anatomically not amenable to embolization or stent grafting.

\section{Case Report}

The patient is a 75 -year-old male with a T1N0M0 pancreatic neuroendocrine tumor who was resectable based on imaging criteria and wished to undergo surgical resection rather than lifelong surveillance. He was offered a robotic Whipple procedure. The technique used is a standardized technique at our institution as has been previously published [1]. Patient's immediate post-operative course was significant for delayed gastric emptying and pancreatic leak. On post-operative day 16, he developed new onset acute blood loss anemia and sudden hypotension. He was emergently transfused with packed red blood cells (PRBC) and fresh frozen plasma (FFP); a nasogastric tube was inserted, and a computerized tomography based angiography (CTA) was performed (Fig. 1). No blood was suctioned via the nasogastric tube. Based on findings of the CTA, the culprit vessel was presumed to be a branch of the superior mesenteric artery (SMA). We proceeded with an angiographyguided localization and attempted embolization of the culprit vessel by IR.

The IR procedure began with access to the right common femoral artery. A Cobra catheter was used to select the celiac artery and a celiac angiogram was performed. No signs of hemorrhage were noted from the branches of the celiac artery. 


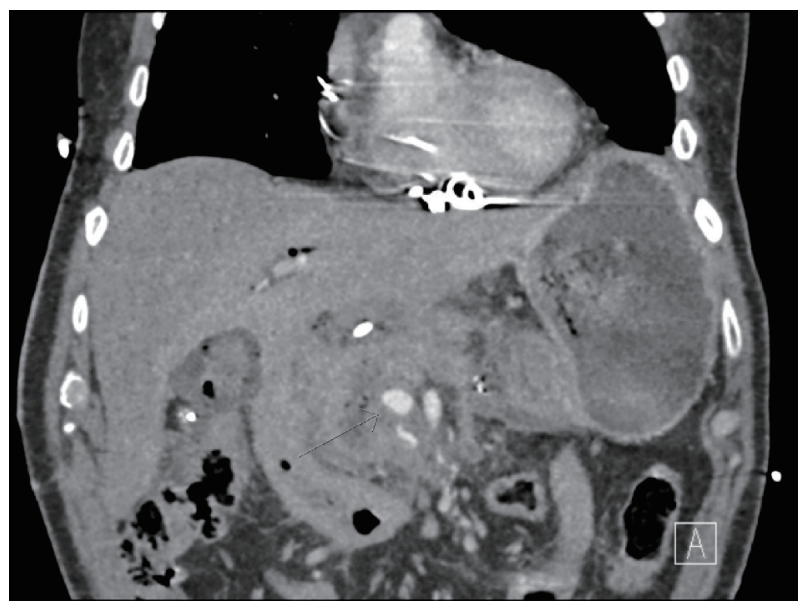

Figure 1. Computed tomography (CT) angiogram showing a "blush" to the right of the superior mesenteric artery (SMA, thin arrow).

This catheter was then withdrawn and used to select the SMA. The SMA angiogram showed a "blush" originating proximally to the right of the SMA (Fig. 2). This proximal vessel could not be cannulated with a micro-catheter despite multiple attempts and angiograms via other branches did not reveal any extravasation. Due to the inability to select the culprit vessel and ongoing hemorrhage, the decision was made to continue resuscitation of the patient and leave a catheter as close to the culprit vessel as possible. Intra-arterial infusion of vasopressin at $0.2 \mathrm{U} / \mathrm{min}$ was initiated via this catheter. Patient was transported to the intensive care unit (ICU) for close monitoring and serial abdominal exams. Over the next $24 \mathrm{~h}$, resuscitation of the patient was continued. The patient had decreasing PRBC requirements and showed no signs of mesenteric ischemia.

A repeat computed tomography $(\mathrm{CT})$ angiogram was per-

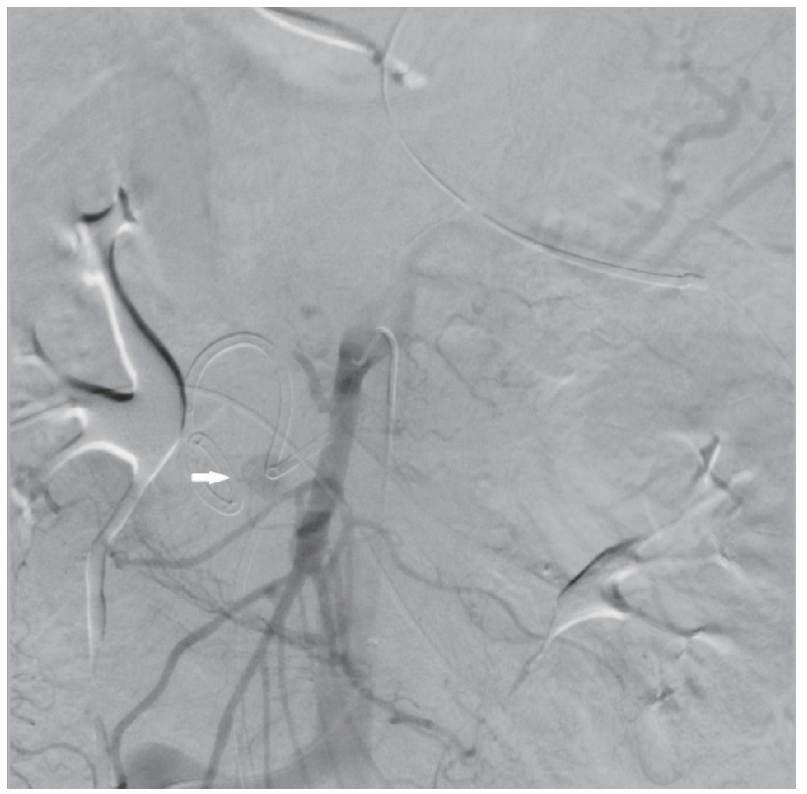

Figure 2. Superior mesenteric artery (SMA) angiogram showing extravasation (thick white arrow).

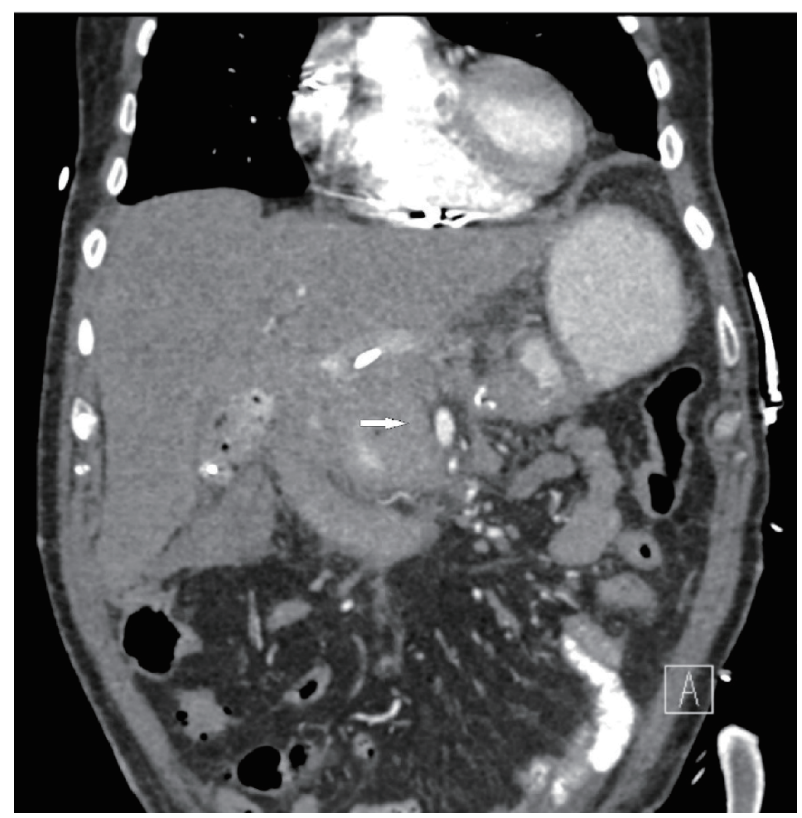

Figure 3. Repeat computed tomography (CT) angiogram showing no further evidence of extravasation from the superior mesenteric artery (SMA, thick white arrow).

formed at $24 \mathrm{~h}$ from last via the pre-existing femoral access. There was no further evidence of bleeding (Fig. 3). The catheter and sheath were removed. The patient was then transferred back to the ICU in stable condition. No subsequent re-bleeding occurred.

\section{Discussion}

In this article we discuss the utility of intra-arterial vasopressin infusion in controlling PPH that is not amenable to more traditional methods of endoscopic or IR interventions. While not considered one of the first-line modalities, intra-arterial vasopressin finds a role in those cases where bleeding is caused either by diffuse lesions or if super-selective catheterization does not allow access to the culprit lesion [2]. Most centers have adopted an algorithm for the management of PPH which begins with endoscopy or interventional angiography for early $\mathrm{PPH}$. These are now considered the first lines of treatment, with redo-laparotomy reserved for those patients who are not responsive to either approach, or for the specific cases of extraluminal bleeding related to technical issues/insufficient hemostasis within the first $24-48 \mathrm{~h}$ [3].

The few cases that are not amenable to control via endovascular embolization, may be considered candidates for intraarterial vasopressin infusion. Once hemorrhage is diagnosed and localized angiographically, attempts are made to obtain control by endovascular embolization. If that fails and the actual site of bleeding is visualized, the $5 \mathrm{~F}$ catheter may be left in the involved vessel. If not, the catheter is secured in the suspected area based on findings from other modalities (e.g., $\mathrm{CT}$ angiography) and the vasopressin infusion is begun. A low, constant rate of $0.1-0.2 \mathrm{U} / \mathrm{min}$ is an appropriate initial dose. If 
this does not control the hemorrhage, the dose may gradually be increased to a maximum of $0.4 \mathrm{U} / \mathrm{min}$, until there is cessation of bleeding. Appropriate vasoconstriction is observed through serial angiograms every $10-15 \mathrm{~min}$. The infusion is then gradually tapered off and replaced with normal saline infusion over the next $24-48 \mathrm{~h}$. The catheter remains in place for yet another $24 \mathrm{~h}$, to observe for possible recurrent bleeding, at which time it is withdrawn. Determinants of technical success include ability to catheterize the main SMA or inferior mesenteric artery (IMA) trunk, atherosclerotic/stenotic lesions preventing appropriate vasospasm and co-existent coagulopathy. Success rates range from $30 \%$ in upper gastrointestinal bleeding to $90 \%$ in lower gastrointestinal bleeding [4]. A systematic search of literature databases did not reveal any prior described use of intra-arterial vasopressin specifically for PPH. Therefore, the rate of success in PPH has not yet been evaluated.

Use of intra-arterial vasopressin infusion is not without risks. In a study by Sherman et al [5], minor complications were found to include hyponatremia, increased urine specific gravity, hypertension, and sinus bradycardia. Major complications included catheter dislodgement ( $2 \%)$, pulmonary edema, significant arrhythmias, and myocardial depression. Another discussion by Conn et al also lists catheter clotting, bleeding from the access site and reactive erythema as minor complications [6]. In their study, a single patient was noted to have necrosis of a significant area of jejunum and ileum related to vasopressin treatment of post-operative stress ulcers, and another patient (cirrhotic) with spontaneous bacterial peritonitis following $24 \mathrm{~h}$ of vasopressin therapy, thought to be due to transmural migration from diminished arterial blood flow to the intestine.

In summary, intra-arterial infusion of vasopressin has been shown to help with PPH which is not amenable to endoscopic or traditional endovascular interventions. Despite falling out of favor over the years, it is imperative that practitioners are familiar with the use, technique, risks and benefits of this method, as it can prove invaluable in challenging clinical situations.

\section{Acknowledgments}

None to declare.

\section{Financial Disclosure}

None to declare.

\section{Conflict of Interest}

The authors have no conflict of interest associated with this article.

\section{Informed Consent}

Informed consent was waived under IRB protocol.

\section{Author Contributions}

All authors have participated sufficiently in the intellectual content, and analysis of data. All authors have reviewed the final version of the manuscript and approve it for publication.

\section{Data Availability}

The authors declare that data supporting the findings of this study are available within the article.

\section{References}

1. Cho E, Pagkratis S, Osman H, Jeyarajah DR. Robotic Pancreaticoduodenectomy. In: Minimally Invasive Surgical Techniques for Cancers of the Gastrointestinal Tract. Springer International Publishing; 2020:123-132.

2. Ramaswamy RS, Choi HW, Mouser HC, Narsinh KH, McCammack KC, Treesit T, Kinney TB. Role of interventional radiology in the management of acute gastrointestinal bleeding. World J Radiol. 2014;6(4):82-92.

3. Wente MN, Shrikhande SV, Kleeff J, Muller MW, Gutt $\mathrm{CN}$, Buchler MW, Friess H. Management of early hemorrhage from pancreatic anastomoses after pancreaticoduodenectomy. Dig Surg. 2006;23(4):203-208.

4. Darcy M. Treatment of lower gastrointestinal bleeding: vasopressin infusion versus embolization. J Vasc Interv Radiol. 2003;14(5):535-543.

5. Sherman LM, Shenoy SS, Cerra FB. Selective intra-arterial vasopressin: clinical efficacy and complications. Ann Surg. 1979;189(3):298-302.

6. Conn HO, Ramsby GR, Storer EH. Selective intraarterial vasopressin in the treatment of upper gastrointestinal hemorrhage. Gastroenterology. 1972;63(4):634-645. 